\title{
Parametric correlation functions to model the structure of permanent environmental (co)variances in milk yield random regression models
}

\author{
A. B. Bignardi, ${ }^{*}$ L. El Faro, † V. L. Cardoso,† P. F. Machado, $\ddagger$ and L. G. Albuquerque $\S^{1}$ \\ *Department of Animal Science, São Paulo State University (FCAV/UNESP), 14884-900, Jaboticabal, SP, Brazil \\ †Agência Paulista de Tecnologia dos Agronegócios - APTA, Pólo Regional Centro Leste, 14075-310, Ribeirão Preto, SP, Brazil \\ łDepartment of Animal Science, University of São Paulo, 13418-900, Piracicaba, SP, Brazil \\ $\S$ Conselho Nacional de Desenvolvimento Científico e Tecnologico (CNPq) and Instituto Nacional de Ciência e Tecnologia - Ciência Animal \\ (INCT- CA), Jaboticabal, SP, Brazil
}

\section{ABSTRACT}

The objective of the present study was to estimate milk yield genetic parameters applying random regression models and parametric correlation functions combined with a variance function to model animal permanent environmental effects. A total of 152,145 test-day milk yields from 7,317 first lactations of Holstein cows belonging to herds located in the southeastern region of Brazil were analyzed. Test-day milk yields were divided into 44 weekly classes of days in milk. Contemporary groups were defined by herd-test-day comprising a total of 2,539 classes. The model included direct additive genetic, permanent environmental, and residual random effects. The following fixed effects were considered: contemporary group, age of cow at calving (linear and quadratic regressions), and the population average lactation curve modeled by fourth-order orthogonal Legendre polynomial. Additive genetic effects were modeled by random regression on orthogonal Legendre polynomials of days in milk, whereas permanent environmental effects were estimated using a stationary or nonstationary parametric correlation function combined with a variance function of different orders. The structure of residual variances was modeled using a step function containing 6 variance classes. The genetic parameter estimates obtained with the model using a stationary correlation function associated with a variance function to model permanent environmental effects were similar to those obtained with models employing orthogonal Legendre polynomials for the same effect. A model using a sixth-order polynomial for additive effects and a stationary parametric correlation function associated with a seventh-order variance function to model permanent environmental effects would be sufficient for data fitting.

Received February 13, 2009.

Accepted May 18, 2009.

${ }^{1}$ Corresponding author: lgalb@fcav.unesp.br
Key words: covariance function, genetic parameter, parametric correlation structure

\section{INTRODUCTION}

Over the past years, random regression models (RRM) have been used for the genetic evaluation of dairy cattle test-day milk yields. These models have the advantage of adjusting milk yields on each test-day, thus permitting the description of specific environmental effects that interfere with milk production, in addition to the estimation of genetic lactation curves for each animal as deviations from the average lactation curve. When employing RRM, there is no need to use adjustment factors for the number of DIM, and these models permit the use of unfinished lactations and the correction for fixed effects that change over time, such as the differences between test months within the same lactation (Swalve, 2000). The results obtained with RRM allow the adoption of selection criteria other than 305-d milk yield, such as milk yields during partial periods of lactation, and persistence of lactation.

One disadvantage of RRM is related to the requirement for computational memory and time for largescale genetic evaluations. Alternative models have been proposed to reduce the number of parameters to be estimated, such as the use of variance functions to model the structure of residual variances and parametric correlation functions to describe the structure of permanent environmental correlations resulting from the animal (Meyer, 2001; Jaffrézic et al., 2002a,b, 2003; Albuquerque and Meyer, 2005). These models are appropriate for cumulative traits, as the observation at specific time is defined as a function of the previous observations.

Nuñez-Antón and Zimmerman (2000) stated that parametric correlation functions present advantages compared with various methods of ANOVA and classical multivariate approach. Usually, they 1) are more efficient for estimating the mean structure and are able to produce more appropriate standard error estimates, 


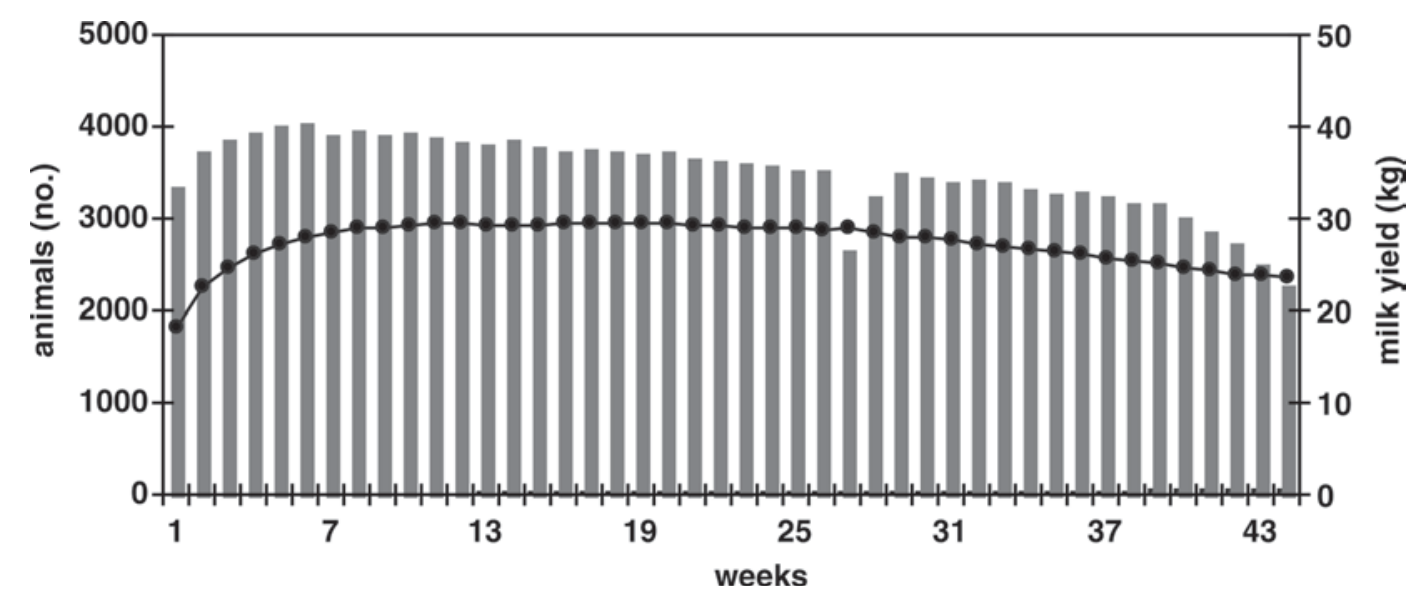

Figure 1. Number of animals (bars) and average test-day milk yields (circles).

2) can deal more effectively with missing data and data measured in different frequency across subjects, and 3) can be employed even when the number of measurements is large relative to the number of subjects.

In the case of stationary parametric correlation functions, it is assumed that the correlation between 2 test records only depends on the difference between DIM on which these 2 records were obtained. In contrast, in the case of nonstationary parametric correlation functions, it is assumed that an observation during time $t$ can be explained by previous observations.

The aim of this paper was to estimate milk yield genetic parameters applying RRM and parametric correlation functions combined with a variance function to model animal permanent environmental effects.

\section{MATERIALS AND METHODS}

A total of 152,145 test-day records from 7,317 first lactations of Holstein cows recorded from 1995 to 2003 were analyzed. Cows were descendants of 612 sires and were distributed over 93 herds located in the southeastern region of Brazil, with age at first calving varying from 18 to 48 mo. Data were obtained from the Herd Analyses and Milk Quality Program carried out by Clínica do Leite (Milk Quality Laboratory of Luiz de Queiroz Agriculture School, ESALQ-USP). Test-day milk yields were recorded from $305 \mathrm{~d}$ of lactation, with the first record being obtained up to $45 \mathrm{~d}$ after calving.

Test-day milk yields were divided into 44 weekly classes of DIM. Lactations with at least 5 individual records were included. Contemporary groups were defined by herd-test-day, with 2,539 groups, and contained at least 6 animals per class. The relationship matrix included 17,688 animals.
Analyses were conducted using a single-trait RRM. The model included the direct additive, permanent environmental, and residual random effects. In addition, contemporary group and linear and quadratic effects of the age of cow at calving were included as fixed effects. The average lactation curve of the population was modeled with a fourth-order orthogonal Legendre polynomial (LP).

Changes in residual variances were modeled by a step function with 6 classes of variance. In this case, the weeks of lactation were grouped as follows: $1,2,3,4$ to 6,7 to 12 , and 13 to 44 . Random additive genetic effects were modeled by random regression on orthogonal LP of DIM. Permanent environmental effects were estimated using a parametric correlation function combined with a variance function. The matrix representation of the model is

$$
\mathbf{y}=\mathbf{X B}+\mathbf{Z} \boldsymbol{\alpha}+\mathbf{W} \boldsymbol{\delta}+\mathbf{e}
$$

where $\mathbf{y}$ is the vector of observations; $\mathbf{B}$ is the vector of fixed effects; $\boldsymbol{\alpha}$ is the vector of additive genetic random coefficients; $\boldsymbol{\delta}$ is the vector of permanent environmental random coefficients; $\mathbf{X}, \mathbf{Z}$, and $\mathbf{W}$ are the correspondent incidence matrices; and $\mathbf{e}$ is the vector of temporary environmental effects. It was assumed that $\mathbf{V}(\boldsymbol{\alpha})$ $=\mathbf{K}_{\mathrm{a}} \otimes \mathbf{A}, \mathbf{V}(\boldsymbol{\delta})=\mathbf{Q}$, and $\mathbf{V}(\mathbf{e})=\mathbf{R}$; the 3 random effects were assumed to be uncorrelated. $\mathbf{V}$ represents variance, $\mathbf{K}_{\mathbf{a}}$ is the (co)variance matrix between regression coefficients for additive genetic effects, $\mathbf{A}$ is the additive numerator relationship matrix, and $\mathbf{Q}$ is the covariance matrix of animal permanent environmental effects. $\mathbf{R}$ represents a diagonal matrix containing residual variances. $\mathbf{Q}$ can be decomposed as

$$
\mathbf{Q}=\sum_{q}^{1 / 2} \mathbf{C} \sum_{q}^{1 / 2}
$$


Table 1. Number of parameters $(p)$, log-likelihood function $(\log L)$, Akaike's information criterion (AIC), and Schwarz's Bayesian information criterion (BIC)

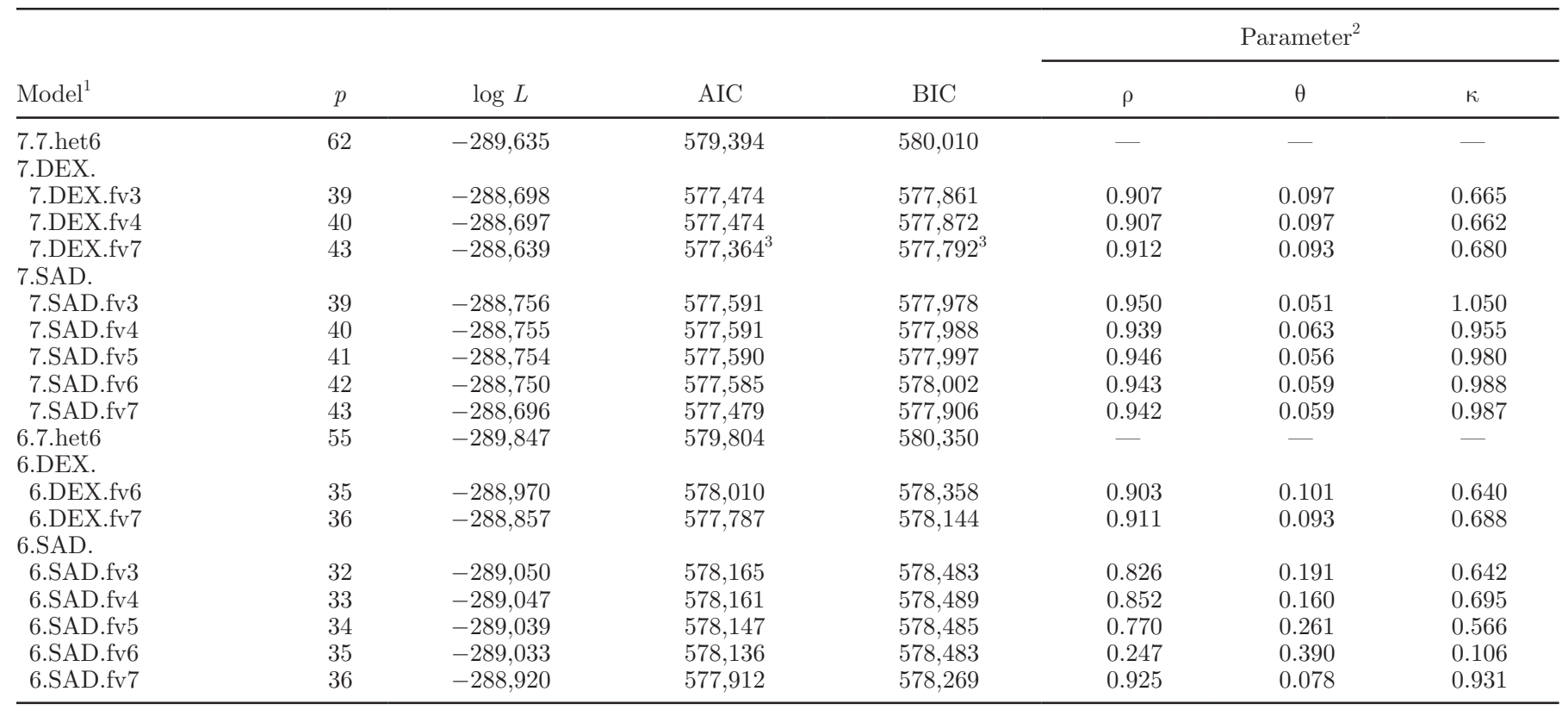

${ }^{1}$ In the form $k_{a} \cdot X .(v f) o$, where $k_{a}=$ order of covariance function fitted to the additive genetic effect, $\mathrm{X}=$ parametric correlation function, and $\mathrm{vf}=$ variance function of order $\mathrm{o}$.

${ }^{2} \rho=$ correlation parameter for structured antedependence model (SAD); $\theta=$ exponential parameter for damped exponential model (DEX); $\kappa$ = scaling parameter for DEX and SAD.

${ }^{3}$ Values indicate the best model based on the AIC and BIC.

with $\sum_{q}=\operatorname{Diag}\left\{\sigma_{q j}^{2}\right\}$ being the diagonal matrix of permanent environmental variances and $\mathbf{C}=\left\{\mathrm{c}_{\mathrm{jk}}\right\}$ being the corresponding matrix of correlations; $\mathbf{C}$ is block diagonal for animals (Meyer, 2001).

Variance of animal permanent environmental effects was modeled using a variance function represented by

$$
\sigma_{j}^{2}=\sigma_{0}^{2}\left\{1+\sum_{r=1}^{v} b_{r}\left(a_{j}^{*}\right)^{r}\right\},
$$

where $\sigma_{j}^{2}$ is the variance on the $j$ th test-day, $\sigma_{0}^{2}$ is the variance on the mean test-day, $b_{r}$ is the coefficient of the variance function, $v$ is the order of the polynomial fit, and $a_{j}^{*}$ is the standardized $(-1$ to +1$)$ age at recording.

Permanent environmental correlations were modeled using a stationary parametric correlation function (damped exponential model; DEX) and a nonstationary correlation function (first-order structured antedependence model; SAD). The variance functions used in the DEX and SAD models were modeled using ordinary polynomials with orders ranging from 3 to 7 .

\section{DEX}

In the case of the stationary correlation function, it is assumed that the correlation between 2 lactation weeks depends only on the difference between DIM on which these 2 records were obtained. There are 2 parameters $(\theta$ and $\kappa)$ that need to be estimated. The model can be described as

$$
r_{i j}=e^{-\theta\left(t_{j}-t_{i}\right)^{\kappa}},
$$

where $r_{i j}=$ correlation between records taken at time $t_{i}$ and $t_{j}, i<j ; \theta$ is the exponential parameter of the autocorrelation; and $\kappa$ is a scaling parameter that permits the attenuation or acceleration of the exponential fall in the autocorrelation function (Meyer, 2001).

\section{$S A D$}

The SAD model, proposed by Nuñez-Antón and Zimmerman (2000), is a nonstationary correlation function. To accommodate nonstationarity, SAD applies a BoxCox transformation to the time scale such as $f\left(t_{i}, k\right)=\left(t_{i}^{k}-1\right) / k$ for $k \neq 0$, and $\log \left(t_{i}\right)$ for $k=0$. Equi- 

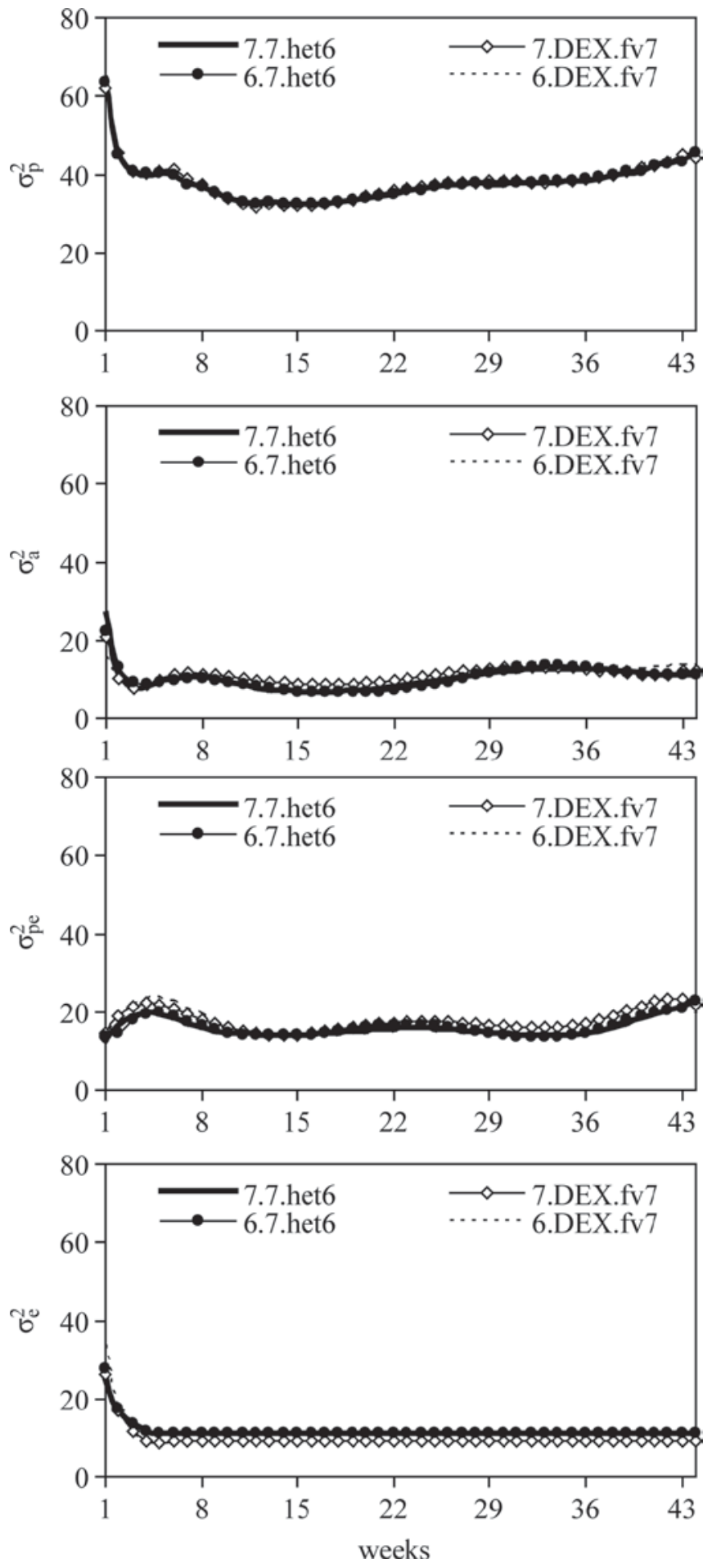

Figure 2. Phenotypic $\left(\sigma_{\mathrm{p}}^{2}\right)$, genetic $\left(\sigma_{\mathrm{a}}^{2}\right)$, permanent environmental $\left(\sigma_{\text {pe }}^{2}\right)$, and residual $\left(\sigma_{\mathrm{e}}^{2}\right)$ variances estimated by the 7.7.het6 and 6.7.het6 (models using a seventh- or sixth-order polynomial for direct additive genetic and seventh-order permanent environmental effects, with the structure of residual variances being modeled with 6 variance classes) and 7.DEX.fv7 and 6.DEX.fv7 (models using a seventh- or sixth-order polynomial for direct additive genetic effect and a parametric correlation function damped exponential, combined with a variance function of seventh order). distant correlations will vary with age if $k \neq 1$, increasing if $k<1$, and decreasing if $k>1$.

(Co)variance components and genetic parameters were estimated by the restricted maximum likelihood method using the DXMRR option of the DFREML statistical software package (Meyer, 1998).

The RRM were compared using Akaike's (AIC) and Schwarz's Bayesian (BIC) information criteria (Wolfinger, 1993), as well as by inspection of the variance and correlation estimates between milk yields on different test-days. The AIC and BIC allow the comparison between nonhierarchical models and penalize those models that contain a larger number of parameters, with the BIC attributing a more rigorous penalty.

The information criteria can be described as

$$
\begin{gathered}
\mathrm{AIC}=-2 \log L+2 p \\
\mathrm{BIC}=-2 \log L+p \log (N-r),
\end{gathered}
$$

where $p$ is the number of parameters in the model, $N$ is the total number of observations, $r$ is the rank of the incidence matrix of fixed effects in the model, and log $L$ is the logarithm of the REML function. Lower AIC and BIC indicate better fit.

Different models are identified as $\mathrm{k}_{\mathrm{a}}$.X.(vf)o, fitting a covariance function of order $\mathrm{k}_{\mathrm{a}}$ to the additive genetic effect and a parametric correlation function X, with X $=\mathrm{DEX}$ or $\mathrm{SAD}$, combined with a variance function (vf) of order o.

The following 2 models employing LP chosen in a previous study were used to compare the results obtained with different RRM (Bignardi et al., 2009): 7.7.het6 [model using a seventh-order polynomial for direct additive genetic $\left(k_{\mathrm{a}}\right)$ and permanent environmental effects $\left(k_{\mathrm{pe}}\right)$, with the structure of residual variance being modeled with 6 variance classes], and 6.7.het6 (model using a sixth- and seventh-order polynomial for $\mathrm{k}_{\mathrm{a}}$ and $\mathrm{k}_{\mathrm{pe}}$, respectively, with the structure of residual variance being modeled with 6 variance classes). In both models, orthogonal LP on DIM was used for both additive genetic and permanent environmental effects.

Random regression coefficient solutions for each sire (612) were used to estimate breeding values for each test-day as proposed by Jamrozik and Schaeffer (1997). Spearman correlations between predicted breeding values obtained with model 6.7.het6 and the 2 best parametric function models, and the coincidence of rank for the 2 and $5 \%$ best sires were calculated.

\section{RESULTS AND DISCUSSION}

Average test-day milk yield was $27.45 \mathrm{~kg}$, with a standard deviation of $7.61 \mathrm{~kg}$ and a coefficient of variation 


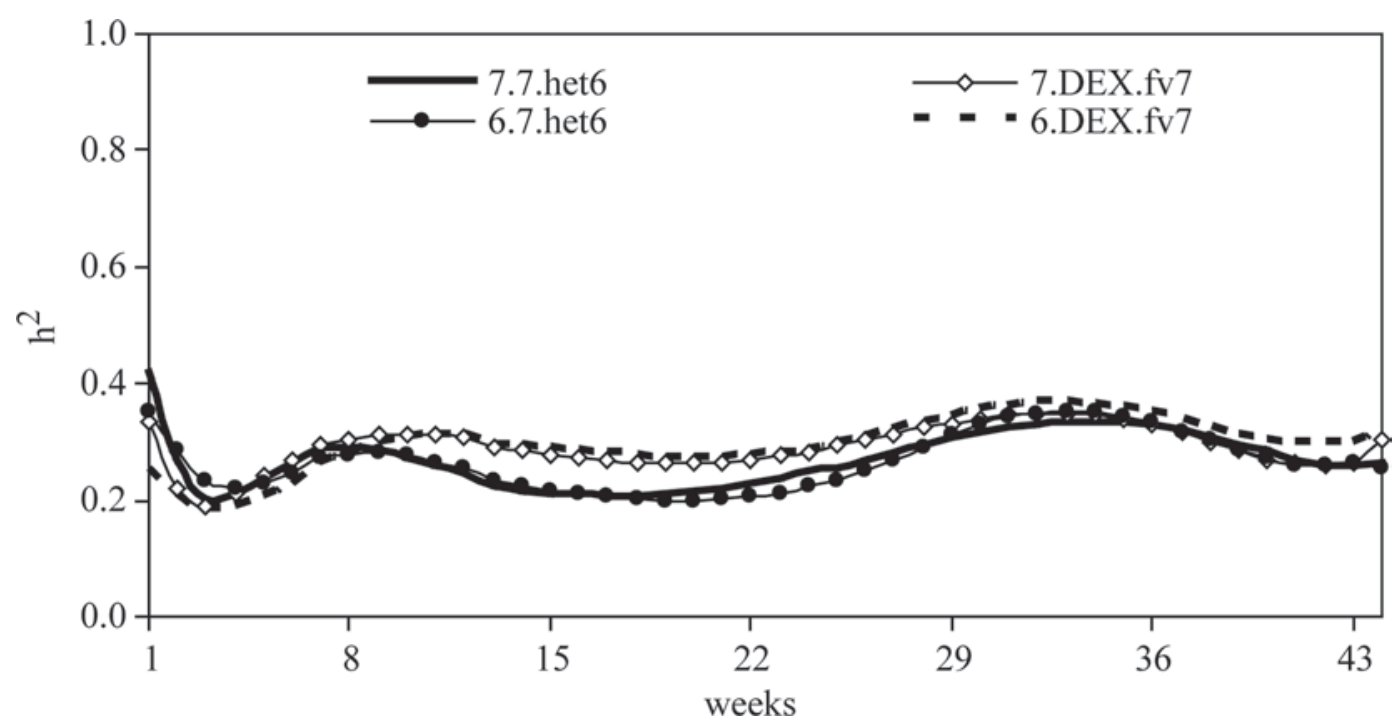

Figure 3. Heritability $\left(\mathrm{h}^{2}\right)$ estimates for weekly test-day milk yield obtained with the 7.7.het6 and 6.7.het6 (models using a seventh- or sixthorder polynomial for direct additive genetic and seventh-order permanent environmental effects, with the structure of residual variances being modeled with 6 variance classes) and 7.DEX.fv7 and 6.DEX.fv7 (models using a seventh- or sixth-order polynomial for direct additive genetic effect and a parametric correlation function damped exponential, combined with a variance function of seventh order).

of $27.73 \%$ (Figure 1). The number of animals decreased at the end of lactation. Discarding contemporary groups with fewer than 6 animals explains the small number of animals in wk 27 and 28 of lactation.

A summary of the results; that is, the value of the likelihood function $(\log L)$, AIC, and BIC is shown in Table 1. Models 7.DEX.fv5, 7.DEX.fv6, 6.DEX.fv3, 6.DEX.fv4, and 6.DEX.fv5 did not reach convergence. The remaining models, considering a parametric correlation function, resulted in higher $\log L$ values and lower AIC and BIC when compared with the 7.7.het6 model. These results suggest that, when using a parametric correlation function, the order of the variance function that is sufficient to describe changes with age will depend on the order of fit for additive genetic effects. Lower orders for additive genetic effects require a higher order variance function for permanent environmental effects.

As expected, the increase in the order of the variance function generally resulted in the increase of log $L$ values. Based on the 2 information criteria, AIC and BIC, the best model consisted of a seventh-order LP for additive genetic effects, a DEX correlation structure associated with a seventh-order variance function to model permanent environmental covariances (7.DEX. fv7).

Jaffrézic et al. (2003) also observed that models using a parametric correlation structure for permanent environmental covariances provided a better fit of data than those using LP. For growth traits of beef cattle, models using both a stationary (Meyer, 2001) and non- stationary (Albuquerque and Meyer, 2005) parametric correlation function provided better fits than RRM employing LP for the same effect.

Similar phenotypic variances were obtained with all models (Figure 2). Genetic, permanent environmental, and residual variances estimated by RRM using LP (6.6.het6 or 7.7.het6) or DEX for permanent environmental effects showed the same trend as the number of DIM increased (Figure 2). Small differences between LP models and models fitting a stationary parametric correlation function (DEX) were observed in terms of the portion of additive genetic and permanent environmental variances. However, when using a nonstationary parametric correlation function (SAD), overestimation of additive variances and underestimation of permanent environmental variances were observed, which resulted in much higher heritability estimates than those obtained with the other models (not shown).

Heritability estimates obtained with the 2 LP models and with the models employing a parametric correlation structure (DEX) presented a similar trend along lactation, with higher estimates being observed at the beginning and the end of lactation (Figure 3). A similar pattern was obtained by Strabel and Misztal (1999) and Brotherstone et al. (2000). Small differences in heritability estimates between the LP models and the models employing a parametric correlation structure were accompanied mainly by differences in residual variances. The estimates ranged from 0.20 to 0.42 (7.7.het6), from 0.20 to 0.35 (6.7.het6), from 0.19 to 0.35 (7.DEX.fv7), and from 0.19 to 0.37 (6.DEX.fv7). These estimates are 

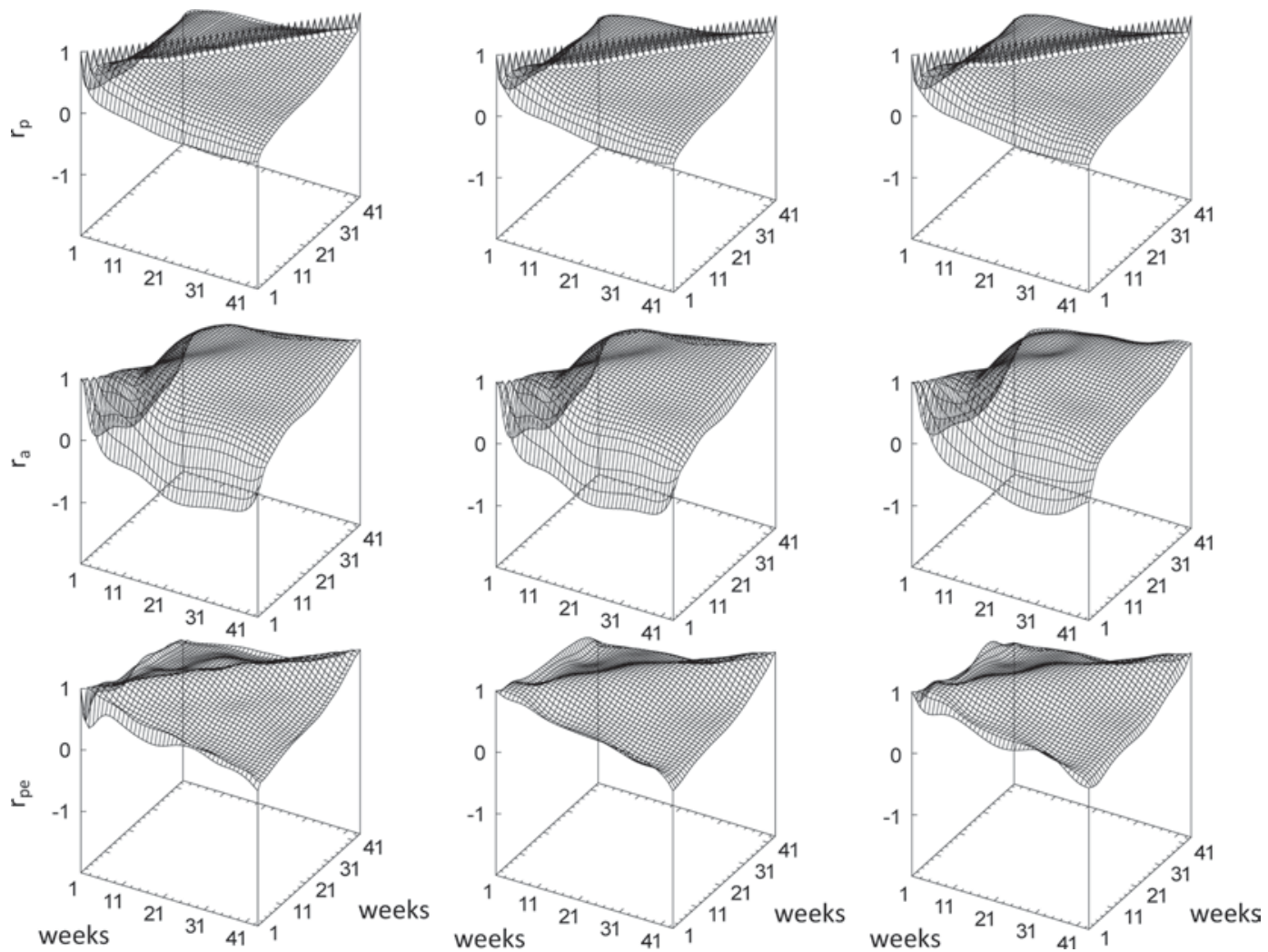

Figure 4. Phenotypic $\left(\mathrm{r}_{\mathrm{p}}\right)$, genetic $\left(\mathrm{r}_{\mathrm{a}}\right)$, and permanent environmental $\left(\mathrm{r}_{\mathrm{pe}}\right)$ correlation estimates (from top to bottom) between weekly milk yields obtained with the 7.7.het6 (first column; model using a seventh-order polynomial for direct additive genetic and seventh-order permanent environmental effects, with the structure of residual variances being modeled with 6 variance classes), 7.DEX.fv7 (second column) and 6.DEX. fv7 (third column; model using a seventh- or sixth-order polynomial for direct additive genetic effect and a parametric correlation function damped exponential, combined with a variance function of seventh order).

close to those reported by Araújo et al. (2006) using orthogonal LP and by Cobuci et al. (2005) using an exponential Wilmink (1987) function.

Phenotypic correlations estimated by the 6.7.het6, 7.DEX.fv7, and 6.DEX.fv7 models presented similar surfaces, with higher values between adjacent test-days during mid lactation and lower values between testdays at the beginning and the end of lactation (Figure 4). Phenotypic correlation estimates between test-days obtained with the 7.DEX.fv7 and 6.DEX.fv7 models ranged from 0.00 to 0.76 and from -0.01 to 0.76 , respectively.

High genetic correlations, close to 1 , were observed between adjacent test-day milk yields (mid lactation). The values decreased as the distance between test-days increased. Similar results were reported by Cobuci et al. (2005). For the 7.DEX.fv7 model, negative genetic correlation estimates were observed between the first test-day and test-days from wk $6(-0.03)$ to wk 43 $(-0.09)$ of lactation, and between the second test-day and test-days from wk $18(-0.03)$ to wk $42(-0.06)$. A similar trend was also observed for the estimates obtained with the 7.7.het6 model. However, a lower frequency of negative genetic correlations was observed with the 6.DEX.fv7 model. Kettunen et al. (2000), Brotherstone et al. (2000), and Cobuci et al. (2005) also reported negative genetic correlation estimates. Negative estimates obtained in the present study could be attributed to difficulties in modeling the initial testdays of the lactation, when the cow is still under the calving stress.

Permanent environmental correlations estimated with the models using a stationary parametric correlation function presented plainer surfaces than those obtained with the LP models. When an LP was used to model permanent environmental effects, oscillations were observed in the correlations between the beginning and the end of lactation, which was not the case when DEX was used for the same effect. According to Meyer (2001), this finding might be attributed to the fact that parametric correlation functions can eliminate erratic behaviors of correlation estimates between measures obtained at extreme ages. The permanent environmental correlations obtained with the 7.7.het6, 7.DEX.fv7, and 6.DEX.fv7 models ranged from 0.24 to 0.99 , from 0.30 to 0.91 , and from 0.28 to 0.91 , respectively. Higher 
values were observed between adjacent test-days and lower values between more distant test-days for all models.

The rank correlation between sire breeding values predicted with models 6.7 .het6 and 7.DEX.fv7; 6.7.het6 and 6.DEX.fv7; and 7.DEX.fv7 and 6.DEX.fv7 were high, at $98.87 \%, 98.70 \%$, and $99.55 \%$, respectively. These results suggest that sires would be ranked practically in the same order independent of the model. Considering sires selected based on model 6.7.het6, the degree of coincidence obtained if selection was based on model 7.DEX.fv7 and 6.DEX.fv7, respectively, were 75 and $66.67 \%$ for the top $2 \%$ (12 sires) and 90.32 and $87.10 \%$ for the top $5 \%$ (30 sires). Therefore, the most parsimonious model (6.DEX.fv7) could be applied for milk yield genetic evaluations using random regressions. The stationary correlation function combined with a variance function to model animal permanent environmental effects in this study was able to model test-day data parsimoniously and appropriately.

Parametric correlation functions seem to be very promising for genetic analysis of repeated measures in the single-trait cases and could be extended to multitrait random regression models, where a dramatic increase in the number of parameters is required to describe complicated covariance structures. Jaffrézic et al. (2003) derived methods for the genetic analysis of 2 or more correlated longitudinal traits assuming structured antedependence functions for both random effects (genetic and permanent environmental).

\section{CONCLUSIONS}

Among the models using parametric correlation structures to model permanent environmental effects, the stationary function (DEX) yielded better results than the nonstationary (SAD) model. Genetic parameter estimates obtained with the model using a stationary correlation function to model permanent environmental effects were similar to those obtained with the model using orthogonal LP for the same effect. The use of a parametric correlation function instead of a polynomial function results in a substantial reduction in the number of parameters necessary to be estimated.

\section{ACKNOWLEDGMENTS}

This study was supported by the State of São Paulo Research Foundation (Fapesp) and National Council of Technological and Scientific Development (CNPq).

\section{REFERENCES}

Albuquerque, L. G., and K. Meyer. 2005. Estimates of covariance functions for growth of Nelore cattle applying a parametric correlation structure to model within-animal correlations. Livest. Prod. Sci. 93:213-222.

Araújo, C. V., R. A. Torres, C. N. Costa, R. A. Torres Filho, S. I. Araújo, P. S. Lopes, A. J. Regazzi, C. S. Pereira, and J. L. R. Sarmento. 2006. Uso de modelos de regressão aleatória para descrever a variação genética da produção de leite na raça Holandesa. R. Bras. Zootec. 35:975-981.

Bignardi, A. B., L. El Faro, L. G. Albuquerque, V. L. Cardoso, and P. F. Machado. 2009. Random regression models to estimate testday milk yield genetic parameters Holstein cows in southeastern Brazil. Livest. Sci. 123:1-7.

Brotherstone, S., I. M. S. White, and K. Meyer. 2000. Genetic modeling of daily yields using orthogonal polynomials and parametric curves. Anim. Sci. 70:407-415.

Cobuci, J. A., R. F. Euclydes, P. S. Lopes, C. N. Costa, R. A. Torres, and C. S. Pereira. 2005. Estimation of genetic parameters for test-day milk in Holstein cows using a random regression model. Genet. Mol. Biol. 28:75-83.

Jaffrézic, F., I. M. S. White, and R. Thompson. 2003. Use of score test as a goodness-of-fit measure of the covariance structure in genetic analysis of longitudinal data. Genet. Sel. Evol. 35:185-198.

Jaffrézic, F., I. M. S. White, R. Thompson, and P. M. Visscher. 2002a. Contrasting models for lactation curve analysis. J. Dairy Sci. 85:968-975.

Jaffrézic, F., I. M. S. White, R. Thompson, P. M. Visscher, and W. G. Hill. 2002b. Statistical models for estimating the genetic analysis of longitudinal data. In Proc. 7th World Congr. Genet. Appl. Livest. Prod., Montpellier, France.

Jamrozik, J., and L. R. Schaeffer. 1997. Genetic evaluation of dairy cattle using test-day yields and random regression model. J. Dairy Sci. 80:1217-1226.

Kettunen, A., E. A. Mäntysaari, and J. Pösö. 2000. Estimation of genetic parameters daily milk yield of primiparous Ayrshire cows by random regression test-day models. Livest. Prod. Sci. 66:251261.

Meyer, K. 1998. DXMRR - A program to estimate covariance functions for longitudinal data by restricted maximum likelihood. In Proc. 6th World Congr. Genet. Appl. Livest. Prod. University of New England, Armidale, NSW, Australia. 27:465-466.

Meyer, K. 2001. Estimating genetic covariance functions assuming a parametric correlation structure for environmental effects. Genet. Sel. Evol. 33:557-585.

Nuñez-Antón, V., and D. L. Zimmerman. 2000. Modeling nonstationary longitudinal data. Biometrics 56:699-705.

Strabel, T., and I. Misztal. 1999. Genetic parameters for first and second lactation milk yields of Polish Black and White cattle with random regression test-day models. J. Dairy Sci. 82:2805-2810.

Swalve, H. H. 2000. Theoretical basis and computational methods for different test-day genetic evaluation methods. J. Dairy Sci. $83: 1115-1124$.

Wilmink, J. B. M. 1987. Adjustment of test-day milk, fat and protein yields for age, season and stage of lactation. Livest. Prod. Sci. 16:335-348.

Wolfinger, R. D. 1993. Covariance structure selection in general mixed models. Commun. Stat. Simul. Comput. 22:1079-1106. 